\title{
DELIRIUM IN ELDERLY PATIENTS UNDERGOING CHEMOTHERAPY FOR SOLID CANCERS AND LYMPHOMA
}

\author{
Y. Kirkova1,2, Y.J. Hwang ${ }^{3}$
}

1 University Hospitals Cleveland Medical Center, Geriatrics and Palliative Care, Cleveland, OH, USA

${ }^{2}$ Medstar Georgetown University Hospital, Palliative Medicine, Washington DC, USA

${ }^{3}$ Case Western Reserve University School of Medicine, Medicine, Cleveland, OH, USA

\section{INTRODUCTION}

Delirium in geriatric cancer patients is poorly understood.

\section{OBJECTIVES}

To determine the incidence and factors associated with delirium in elderly patients undergoing chemotherapy for solid cancers.

\section{METHODS}

A retrospective chart review of consecutive cancer patients $\geq 65$ y old.

- Main objective was to identify 20 individuals with solid tumors and lymphomas on chemotherapy or chemo-radiation during 2015.

- Admissions within 30 days of anti-cancer treatment were included.

口 Admitting diagnosis included: "Delirium", "Encephalopathy",

"Confusion", "Altered mental status".

$\square$ Statistical analysis:

Descriptive and matched pair statistics compared demographics, chemotherapy, medications, comorbidities, and laboratory data between the identified patients and their controls matched by age, gender, and cancer diagnosis.

\section{RESULTS}

$\square \mathrm{N}=123$ patients hospitalized in an academic comprehensive cancer center at Cleveland Medical Center, Cleveland, $\mathrm{OH}$.

$\square$ Demographics and cancer type are in Table 1.

Delirium incidence was $7.3 \%(N=9 / 123)$. $N=5$ were men

\section{RESULTS cont.}

The most common hospital admissions were: chemotherapy (22\%) and infection (21\%).

- 64\% received, $24 \%$ - chemotherapy and immunotherapy, and $12 \%$ immunotherapy.

- $43 \%$ were treated with combinations of 2 agents, and $32 \%$ with a single agent only.

Compared to matched controls, patients with delirium had a higher BUN, more acute kidney injury (AKI defined as $>30 \%$ increase in baseline creatinine) and hyponatremia (Fig.1).

- There were trends in worse ECOG PS, lower Hct, Hgb, elevated ALT, AST, and more CAD, CKD stage III, depression, diabetes and UTI.

\section{CONCLUSIONS}

Low delirium incidence in cancer patients $\geq 65$ years old within 30 days of chemotherapy was most likely due to delirium underreporting and excellent patient selection prior to chemotherapy.

$\square$ The most common admission reasons were infections and chemotherapy.

Elevated BUN, AKI and hyponatremia were significantly associated with delirium incidence.

Trends in worse ECOG, anemia, transaminitis and more comorbidities were observed.

\begin{tabular}{|c|c|c|}
\hline Patients & $N=123$ & $100 \%$ \\
\hline Age, Median (range) & $71(65-89)$ & NA \\
\hline Gender, Females & 74 & 60 \\
\hline \multicolumn{3}{|l|}{ Cancer type } \\
\hline Gastrointestinal & 32 & 25 \\
\hline Colorectal & $13 / 32$ & \\
\hline Esophageal, Stomach, Hepatobiliary & $11 / 32$ & \\
\hline Pancreas & $8 / 32$ & \\
\hline Lymphoma & 24 & 20 \\
\hline Genitourinary & 22 & 18 \\
\hline Ovary, Uterine & $11 / 22$ & \\
\hline Prostate & $5 / 22$ & \\
\hline Renal, Bladder, Testis & $6 / 22$ & \\
\hline Lung & 18 & 15 \\
\hline Breast & 15 & 12 \\
\hline Head and Neck & 6 & 5 \\
\hline Other (Peritoneal, Melanoma, Sarcoma) & 6 & 5 \\
\hline
\end{tabular}

\begin{tabular}{|c|c|c|c|c|c|c|c|}
\hline $\begin{array}{l}\text { Age } \\
\text { D }\end{array}$ & $\begin{array}{l}\text { Age } \\
\text { C }\end{array}$ & $\begin{array}{l}\text { Gender } \\
\text { D, C }\end{array}$ & $\begin{array}{l}\text { Cancer Type } \\
\text { D/C }\end{array}$ & $\begin{array}{l}\text { Admission } \\
\text { Cause D }\end{array}$ & $\begin{array}{l}\text { Admission } \\
\text { Cause C }\end{array}$ & $\begin{array}{l}\text { Treatment } \\
\text { D }\end{array}$ & $\begin{array}{l}\text { Treatment } \\
\mathrm{C}\end{array}$ \\
\hline 74 & 71 & $\mathbf{M}$ & Lymphoma & Sepsis & $\begin{array}{l}\text { Bacteremia } \\
\text { Abscess }\end{array}$ & $\mathrm{Ch}+$ & $\mathrm{Ch}+\mathrm{I}$ \\
\hline 69 & 68 & $\mathbf{M}$ & Lymphoma & Seizure, Sepsis & Ch & $\mathrm{Ch}+$ & $\mathrm{Ch}+\mathrm{I}$ \\
\hline 65 & 66 & $\mathbf{M}$ & Lymphoma & $\begin{array}{l}\text { Enc, } \\
\text { Hyperbilirubinemia }\end{array}$ & L1 spinal mass & $\mathrm{Ch}+$ & Ch \\
\hline 66 & 73 & $\mathbf{F}$ & Lymphoma & Enc & Ch & $\mathrm{Ch}+$ & $\mathrm{Ch}+\mathrm{I}$ \\
\hline 68 & 73 & $\mathrm{~F}$ & Lung & Enc, Fever & Ch-Radiation & $\mathrm{Ch}$ & $\mathrm{Ch}$ \\
\hline 68 & 67 & $\mathbf{M}$ & Lung & Dehydration & Hemoptysis & $\mathrm{Ch}$ & Ch \\
\hline & & & & & Hypoxia, & & \\
\hline 65 & 67 & $\mathrm{~F}$ & Lung & Enc & COPD & $\mathrm{Ch}$ & Ch \\
\hline 72 & 71 & M & $\begin{array}{l}\text { Pancreas/ } \\
\text { Stomach } \\
\end{array}$ & Ch & $\begin{array}{l}\text { Respiratory } \\
\text { failure }\end{array}$ & $\mathrm{Ch}$ & $\mathrm{Ch}$ \\
\hline 71 & 70 & $\mathbf{F}$ & $\begin{array}{l}\text { Eso, Pancreas/ } \\
\text { Pancreas }\end{array}$ & $\begin{array}{l}\text { Hypoglycemia, } \\
\text { Anorexia }\end{array}$ & GI bleed & $\mathrm{Ch}$ & Ch \\
\hline
\end{tabular}

Fig. 1. Comparison Between Serum Na and Serum Urea (BUN) in Patients with Delirium (D) and Matched Controls (C)
Serum $\mathrm{Na}$ and BUN in Patients with Delirium (D) vs. Controls (C)

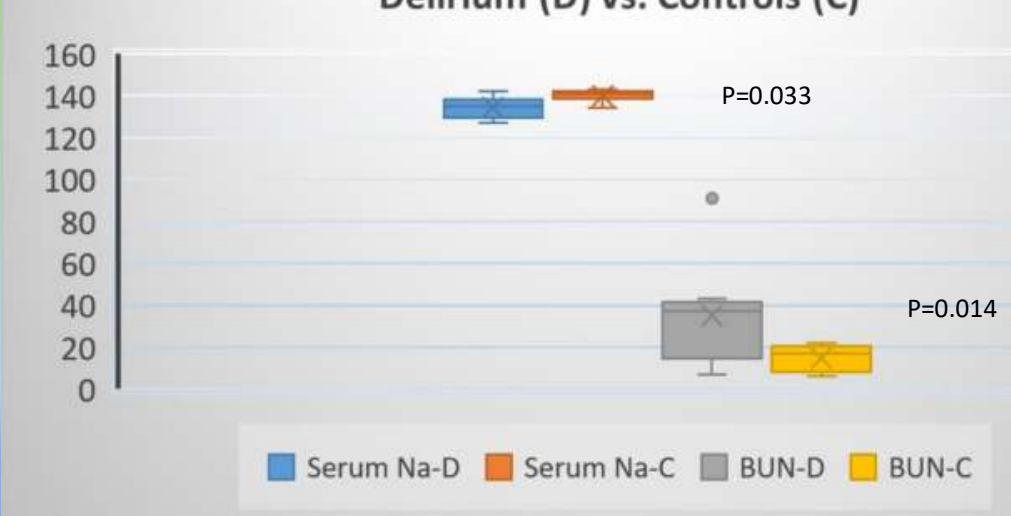

